\title{
CVD synthesis of carbon spheres using NiFe-LDHs as catalytic precursors: structural, electrochemical and magnetoresistive properties
}

\author{
Jose A. Carrasco ${ }^{a}$, Helena Prima-Garcia ${ }^{a}$, Jorge Romero ${ }^{a}$, Jesús Hernández- \\ Saz ${ }^{b}$, Sergio I. Molina ${ }^{b}$, Gonzalo Abellán ${ }^{\star_{a c}}$ and Eugenio Coronado ${ }^{*}$ \\ anstituto de Ciencia Molecular (ICMol), Universidad de Valencia, Catedrático José \\ Beltrán 2, 46980, Paterna, Valencia, Spain. E-mail: eugenio.coronado@uv.es; Fax: +34 \\ 96354 3273; Tel: +34 963544415 \\ 'Departamento de Ciencia de los Materiales e Ingeniería Metalúrgica y Química \\ Inorgánica, IMEYMAT, Universidad de Cádiz, 11510 Puerto Real, Cádiz, Spain \\ 'Department of Chemistry and Pharmacy and Institute of Advanced Materials and \\ Processes (ZMP), University Erlangen-Nürnberg, Henkestr. 42, 91054 Erlangen and Dr.- \\ Mack Str. 81, 90762 Fürth, Germany. E-mail: gonzalo.abellan@fau.de; Fax: +49 911 \\ 65078 65015; Tel: +499116507865031
}

\section{Abstract}

The gram-scale synthesis of carbon spheres with a diameter of $c a .740 \mathrm{~nm}$ has been achieved by means of a chemical vapour deposition method using NiFe-layered double hydroxides as a solid catalytic precursor. The presence of the catalyst $\left(\mathrm{FeNi}_{3}\right)$ allows controlling the final size distribution, resulting in a monodisperse sample. Their structural properties exhibited a high degree of graphitization according to their $I_{D} / I_{G}$ ratio. In addition, their morphological features were unveiled by FIB-SEM and HRTEM, showing that they are formed by solid inner cores, and presenting labile chain-like structures due to accretion procedures. The solution and posterior sonication of the samples in toluene gave rise to the well-defined isolated spheres. The textural and electrochemical properties of the spheres have been tested showing non-mesoporous structures with a good behaviour as electrode materials for 
supercapacitors due to the presence of redox functionalities on their surface. Finally, magneto-transport measurements have been carried out, demonstrating semiconductor behaviour, as well as a positive magnetoresistance effect (ca. $72 \%$ ) for the lowest studied temperature $(2 \mathrm{~K})$.

\section{Introduction}

Since the discovery of fullerenes $\left(C_{60}\right)$ by Kroto et $a l$. in $1985,{ }^{1}$ many studies have been published in the field of the so-called carbon nanoforms (CNF). These carbon-based materials include a wide variety of sizes and shapes ranging from $C_{60}$ to metallofullerenes, carbon fibers, nanotubes (single-wall, multi-wall...), nano-onions, nanohorns, nanobuds, spheres or graphene, among others. ${ }^{2}$ The interest in different synthetic carbon allotropes relies on their outstanding properties and applications, as recently highlighted by the ubiquitous graphene. Among this family, spherical carbon structures like $\mathrm{C}_{60}$, or carbon nano-onions have attracted increasing attention in the last few years. One of these forms are carbon spheres (CS), formerly known as carbon blacks. ${ }^{3}$ The curved structure of CS is a consequence of the presence of pentagonal and heptagonal rings within the hexagonal framework of carbon, allowing the system to adopt their characteristic morphology. ${ }^{4}$

Carbon spheres can be classified according to different parameters, like their size, nanometric texture or internal structure. ${ }^{5-8}$ Recently, Deshmukh et al. reviewed different techniques employed for the synthesis of CS, pointing out chemical vapour deposition (CVD) as one of the most versatile synthetic approaches. ${ }^{9}$

These carbonaceous forms show a wide range of potential applications due to their thermal stability, electronic properties, high surface area or low density. Furthermore, their unclosed graphitic flakes give rise to dangling bonds throughout the surface of the spheres, increasing their reactivity and boosting their applicability in adsorption, lubricants, tribological coatings, catalysis, batteries, supercapacitors or fuel cells among others. ${ }^{10-15}$ Conversely, when it comes to their transport and magnetic properties, the list of examples is very scarce,,$^{16,17}$ despite their potential for use as electronically active reinforcing agents of functional composites..$^{18,19}$ Indeed, the recent discovery of organic magnetoresistance (OMAR) in graphene and other related nanocarbons has significantly attracted increasing attention to these systems, as they are promising materials for flexible, low-cost and transparent electronics. ${ }^{20}$ 
${ }^{25}$ Therefore, the cost-effective production of magnetoresistive CS is of great interest in the development of new hybrid composites and functional polymers.

Herein, we have synthesized monodisperse carbon spheres in a gram-scale by means of a CVD procedure using NiFe-layered double hydroxides (LDHs) as catalytic precursors. ${ }^{26}$ The NiFe-LDH materials have demonstrated their utility as catalysts in the low-temperature synthesis of several carbon nanoforms, including carbon nanoonions, ${ }^{27}$ bamboo-like carbon nanotubes ${ }^{28}$ or even graphene - when they are used as precursors for the preparation of hybrid $\mathrm{FeNi}_{3}$-graphene nanocomposites. ${ }^{28-30}$

We have thoroughly characterized the as-obtained spheres by means of XRD, Raman, FT-IR, TG, FESEM, FIB-SEM, HRTEM, DLS, $\mathrm{N}_{2}$ isotherms, XPS and Electrochemistry. Furthermore, their magneto-transport properties revealed a semiconducting behaviour with an intrinsic large MR of $c a .72 \%$.

\section{Experimental methods}

\subsection{Chemicals}

All chemical reagents $\mathrm{Ni}\left(\mathrm{NO}_{3}\right)_{2} \cdot 6 \mathrm{H}_{2} \mathrm{O}$ (Aldrich); $\mathrm{Fe}\left(\mathrm{NO}_{3}\right)_{3} \cdot 9 \mathrm{H}_{2} \mathrm{O}$ (Sigma-Aldrich); triethanolamine (TEA), $\mathrm{C}_{6} \mathrm{H}_{15} \mathrm{NO}_{3}$ (Sigma-Aldrich); urea, $\mathrm{CO}\left(\mathrm{NH}_{2}\right)_{2}$ (Fluka) and ethanol absolute (Panreac, 99.9\%) were used as received without further purification. Ultrapure water was obtained from Millipore Milli-Q equipment. $\mathrm{N}_{2}, \mathrm{H}_{2} / \mathrm{Ar}(10 \%)$ and ethylene $\left(\mathrm{C}_{2} \mathrm{H}_{4}\right)$ were purchased from Linde Material Handling.

\subsection{Synthesis of $\mathrm{NiFe}-\mathrm{CO}_{3} \mathrm{LDHs}$}

$\mathrm{NiFe}-\mathrm{CO}_{3} \mathrm{LDHs}$ were synthesized following a modified homogeneous precipitation method by using urea and TEA as an ammonium releasing reagent (ARR) and a chelating agent, respectively. ${ }^{31}$

In a typical synthesis, the nitrate salts of the metals were dissolved in $50 \mathrm{~mL}$ of deionized pure water together with TEA, in order to reach a total metal cation concentration of $20 \mathrm{mM}$ in the final solution, with a $\mathrm{Ni}$ : Fe ratio of $3: 1(x=0.25$, with $\left.x=\left[\mathrm{Fe}^{3+}\right] /\left(\left[\mathrm{Fe}^{3+}\right]+\left[\mathrm{Ni}^{2+}\right]\right)\right)$, and a concentration of TEA equimolar with the iron concentration ( $5 \mathrm{mM})$. Next, $50 \mathrm{~mL}$ of aqueous solution of urea ( $35 \mathrm{mM}$ ) were added. The excess of urea was fixed to be 1.75 times the total metal concentration. The resulting dark brown mixture was placed in a $125 \mathrm{~cm}^{3}$ stainless steel Teflon-lined autoclave and heated up to $150{ }^{\circ} \mathrm{C}$ in a previously heated oven. After $48 \mathrm{~h}$, the 
autoclave was left to cool down at room temperature and the final yellowish fine powder was filtered and dried under a vacuum. The $\mathrm{pH}$ value of the remaining solution was found to be around 8 .

\subsection{Synthesis of carbon spheres}

Carbon spheres were synthesized by means of a chemical vapour deposition process (CVD). In a typical procedure, $25 \mathrm{mg}$ of NiFe-LDHs were placed at the centre of a horizontal quartz tube, which was inserted into a tubular furnace at atmospheric pressure. The furnace was heated under flowing $\mathrm{N}_{2}\left(100 \mathrm{~mL} \mathrm{~min}^{-1}\right)$ up to $900{ }^{\circ} \mathrm{C}$ at a 5 ${ }^{\circ} \mathrm{C}$ min $^{-1}$ scan rate. When the temperature was reached, reduction with $\mathrm{H}_{2}\left(50 \mathrm{~mL} \mathrm{~min}^{-1}\right.$, mixture of $\mathrm{H}_{2} 10 \%$ in $\mathrm{Ar}$ ) was performed for 5 min before switching the gas for the carbon source, ethylene $\left(\mathrm{C}_{2} \mathrm{H}_{4}, 100 \mathrm{~mL} \mathrm{~min}^{-1}\right)$, during 60 more min at $900^{\circ} \mathrm{C}$. Then, the furnace was cooled to room temperature under $\mathrm{N}_{2}$ protection, and the sample was collected from the quartz tube. Control experiments in the absence of NiFe-LDHs were also carried out under the same experimental conditions.

\subsection{Thermal treatment}

The as-synthesized carbon spheres were annealed at $800{ }^{\circ} \mathrm{C}$ in order to eliminate organic impurities. In a typical procedure, the sample was placed in a holder at the centre of a horizontal quartz tube inserted into a furnace at atmospheric pressure. The furnace was heated up to $800{ }^{\circ} \mathrm{C}$ under a $\mathrm{N}_{2}$ atmosphere $\left(100 \mathrm{~mL} \mathrm{~min} \mathrm{~m}^{-1}\right)$ at a $2{ }^{\circ} \mathrm{C}$ $\mathrm{min}^{-1}$ scan rate. When the temperature was reached, the system was cooled to room temperature under $\mathrm{N}_{2}$ protection. Finally, the annealed spheres were collected from the holder.

\subsection{Physical characterization}

X-ray powder diffraction (XRPD) patterns were obtained using a PANalytical Empyrean $\mathrm{X}$-ray powder diffractometer under copper radiation ( $\mathrm{Cu}-\mathrm{Ka}=1.54178 \AA$ ). Samples were mounted on a flat sample plate. Profiles were collected in the $2.5^{\circ}<2 \otimes<90^{\circ}$ range with a step size of $0.05^{\circ}$. Raman measurements (Jobin-Yvon LabRam HR 800 Raman Microscope) were carried out at room temperature with the $532 \mathrm{~nm}$ line of an Ar ion laser as an excitation source. The morphology of spheres was studied by means of field emission scanning electron microscopy (FESEM) performed on a Hitachi S4800 microscope operating at an accelerating voltage of $20 \mathrm{kV}$ over metallized samples with a mixture of gold and palladium for $30 \mathrm{~s}$. FEI dual-beam FIB-SEM Quanta $2003 \mathrm{D}$ equipment operating at $30 \mathrm{kV}$ was used to mill the spheres and observe their inner structure and prepare lamellae by using an in situlift-out method. ${ }^{32} \mathrm{High}$ resolution transmission electron microscopy (HRTEM) studies were carried out on Tecnai G2 F20 and Jeol 2100 microscopes operating at 200 kV. Samples were prepared by dipping a sonicated suspension of the sample in toluene on a carbon-coated copper grid. Metallic atomic composition of bulk samples was determined by means of electron probe microanalysis (EPMA) performed on a Philips SEM-XL30 equipped 
with an EDAX microprobe. Carbon, nitrogen and hydrogen contents were determined by microanalytical procedures by using a LECO CHNS. Infrared spectra were recorded on a FT-IR Nicolet 5700 spectrometer in the $4000-400 \mathrm{~cm}^{-1}$ range using powdered samples diluted in $\mathrm{KBr}$ pellets. Thermogravimetric analysis of the compounds were carried out using a Mettler Toledo TGA/SDTA 851 apparatus in the $25-800{ }^{\circ} \mathrm{C}$ temperature range at a $10{ }^{\circ} \mathrm{C} \mathrm{min}^{-1} \mathrm{scan}$ rate and under an air flow of $30 \mathrm{~mL} \mathrm{~min}{ }^{-1}$. The porous texture was characterized by $\mathrm{N}_{2}$ adsorption at $77 \mathrm{~K}$ in an AUTOSORB-6 apparatus. The samples were previously degassed for $4 \mathrm{~h}$ at $523 \mathrm{~K}$ and $5 \times 10^{-5}$ bar. Dynamic light scattering (DLS) measurements were recorded at $25^{\circ} \mathrm{C}$ using a Zetasizer Nano ZS instrument (Malvern Instrument Ltd) on a freshly prepared sample (1 $\mathrm{mg}$ of $\mathrm{CS}$ in $10 \mathrm{~mL}$ of toluene and sonicated for $30 \mathrm{~min}$ ). The electrical conductivity and magneto-resistance measurements were carried out by using a standard four-probe contact method with Pt wire in a physical property measurement system (PPMS-9) by Quantum Design. The resistivity data were taken from 300 to $2 \mathrm{~K}$ and from 0 to $8 \mathrm{~T}$, with Keithley 2450 as a current source and a Keitley 6514 as an electrometer. The measurements were performed in a pressed pellet of the CS with a size of $2.4 \times$ $10^{-4} \mathrm{~cm}^{2}$

\subsection{Electrochemical measurements}

The electrochemical analyses were performed using an Autolab electrochemical workstation (PGSTAT-100 potentiostat/galvanostat) connected to a personal computer that uses GPES electrochemical software.

The materials were mixed with acetylene black and PVDF at a mass ratio of $80: 10$ : 10 in ethanol and deposited on a nickel foam electrode. The as-prepared nickel foam electrodes were dried overnight at $70{ }^{\circ} \mathrm{C}$ and pressed. Each working electrode contained about $1 \mathrm{mg}$ of electroactive material and had a geometric surface area of about $1 \mathrm{~cm}^{2}$. A typical three-electrode experimental cell equipped with a stainless steel plate having $4 \mathrm{~cm}^{2}$ of surface area as the counter electrode and a Metrohm $\mathrm{Ag} / \mathrm{AgCl}(3 \mathrm{M} \mathrm{KCl})$ as the reference electrode were used for the electrochemical characterization of the materials trapped by working electrodes.

The electrochemical measurements were carried out in aqueous $6 \mathrm{M} \mathrm{KOH}$ solutions as the electrolyte. Ultrapure water was obtained from Milli-Q equipment. The specific capacitance $(C)$ was calculated from the cyclic chronopotentiometric curves according to eqn (1):

$C=I \Delta t / m \Delta V$

where $l$ is the charge/discharge current, $\Delta t$ is the time for a full charge or discharge, $m$ is the mass in grams of the active material in the electrode layer, and $\Delta V$ is the voltage change after a full charge or discharge. 
Electrochemical impedance spectroscopy measurements were carried out by applying an $\mathrm{AC}$ amplitude of $10 \mathrm{mV}$ in the frequency range of $0.01-10^{6} \mathrm{~Hz}$ at an open circuit potential with the help of an impedance spectrum analyser (GAMRY interface 1000 potentiostat). The electrochemical cell was placed in a Faradaic cage. Data were analysed with the help of Gamry Echem Analyst v. 6.03 software.

\section{Results and discussion}

The synthesis of carbon spheres was achieved by means of a catalytic CVD process at $900{ }^{\circ} \mathrm{C}$ using NiFe-LDHs as catalytic precursors and ethylene as a carbon source for a period of $60 \mathrm{~min}$. After the CVD process, a black solid with metallic brilliance was formed using a quartz tube. The sample was collected from the inner part of the quartz reactor, powdered and submitted to a subsequent annealing process at $800^{\circ} \mathrm{C}$ under an inert atmosphere in order to eliminate possible organic impurities. The final product was a black powder light in weight, hereinafter named as CS. Further characterization of pristine spheres is depicted in the ESI \pm (see SI 1).

As recently reported by our group, NiFe-LDHs are excellent catalysts for the synthesis of carbon nanoforms. ${ }^{26,28}$ Moreover, we have demonstrated that the calcinated NiFe-LDH preserves the structural integrity, with the in situ formed $\mathrm{FeNi}_{3}$ nanoparticles being the main catalytic seeds. ${ }^{27}$ Herein, control experiments consisting of the calcination of NiFe-LDHs at $900{ }^{\circ} \mathrm{C}$, supplying $\mathrm{H}_{2}$ but in the absence of a carbon source, gave rise to a mixture of $\mathrm{NiFe}_{2} \mathrm{O}_{4}, \mathrm{NiO}$ and $\mathrm{FeNi}_{3}$ phases as revealed by XRPD (see SI 2.1, ESI 1 ). However, further experiment with neither $\mathrm{H}_{2}$ nor ethylene depicted the presence of only a mixture of $\mathrm{NiFe}_{2} \mathrm{O}_{4}$ and $\mathrm{NiO}$, concluding that the $\mathrm{FeNi}_{3}$ alloy is the active catalytic species in the CVD reaction, responsible for the growth of carbon nanoforms. Indeed, a low-temperature CVD process at $550{ }^{\circ} \mathrm{C}$ with bulk samples leads to a mixture of carbon nanoforms formed by few-layer graphene, carbon fibers and multi-wall carbon nanotubes (see ref. 28 for further information). According to Li and co-workers, these graphitic structures could evolve towards the formation of carbon spheres for larger times of feeding. ${ }^{33}$ Herein, the presence of the catalyst is reflected in a decrease in the starting synthesis temperature towards ca. $800^{\circ} \mathrm{C}$, allowing the gram-scale formation of monodisperse CS (vide infra). In contrast, carbon sphere control experiments without the presence of a catalyst leads to an increase in the temperature to $900{ }^{\circ} \mathrm{C}$ yielding a lower amount of CS with a 
wide range of dimensions and morphologies, highlighting the crucial role of NiFeLDHs (Fig. SI 2.2, ESI \pm ). ${ }^{34}$

Inagaki ${ }^{6,7}$ reported that the most probable mechanism for the formation of carbon spheres consists of the dissociation of ethylene (carbon source) at $900{ }^{\circ} \mathrm{C}$ into $\mathrm{C}$ and $\mathrm{H}$. After that C starts to polymerize forming the so-called basic structural units (BSUs), whilst methane and hydrogen are also formed. Usually, the arrangement of these BSUs leads to three different textures in these carbon forms: concentric, radial and random. ${ }^{2}$ The final disposition of these units depends on the precursor, heating treatment, feeding time and the interface between the carbon atoms and their surroundings during the thermal process. ${ }^{735,36}$ Anyway, the exact role of the catalyst in the formation of spheres mediated by CVD procedures is not yet fully revealed. It seems that the $\mathrm{FeNi}_{3}$ catalyst may act as a template, providing nucleation sites for the subsequent formation of spheres. Another possibility is its role in the modification and deposition of carbon radicals which are formed in the gas phase, i.e., helping the decomposition of the carbon source instead of the polymerization reaction. ${ }^{\circ}$

The XRPD spectrum in CS was recorded in order to study the graphitic structure of the sample. Fig. $1 \mathrm{~A}$ exhibits the presence of a main peak centred at $c a .25^{\circ}$ and a secondary peak at $c a .43 .8^{\circ}$, related to the (002) and the (100) planes of graphite, respectively. The common broadness of the peaks in the carbon sphere spectra indicates a low range in the structural order. ${ }^{10,28}$ According to Bragg's equation, the interlayer spacing $d_{002}$ of our CS is $0.356 \mathrm{~nm}$, which agrees with the literature, ${ }^{30,31}$ with a low deviation for the ideal $d_{002}$ space $(0.343 \mathrm{~nm})$ in a graphitic structure (JCPDS-ICDD Card No. 41-1487). This value is intermediate between those measured for a carbon fiber $\left(d_{002}=0.342\right)$ and activated carbon atoms $\left(d_{002}=0.363\right)$, which is indicative of a relatively well-organised carbon structure. ${ }^{5}$ 
A

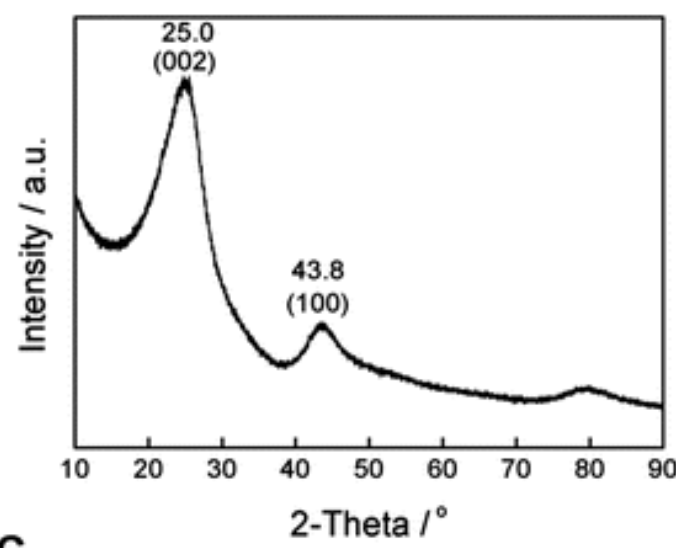

C

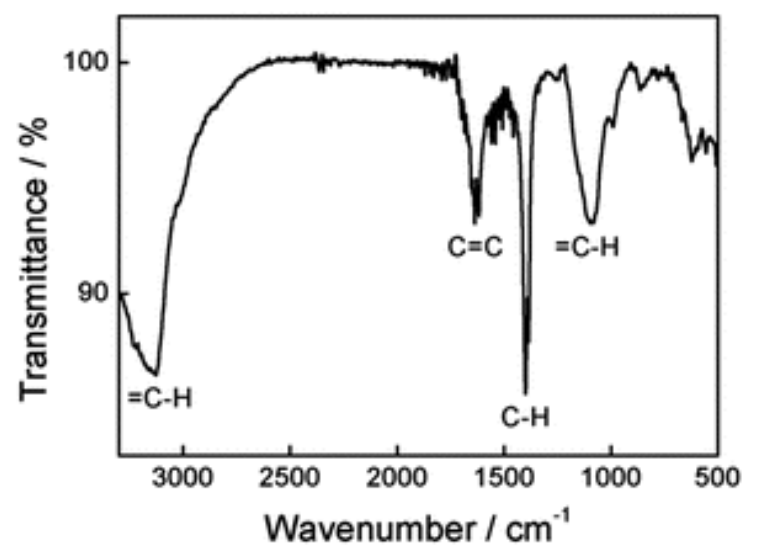

B

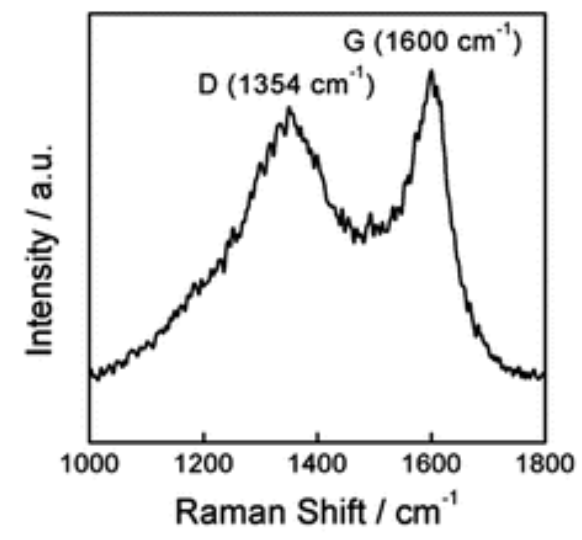

D

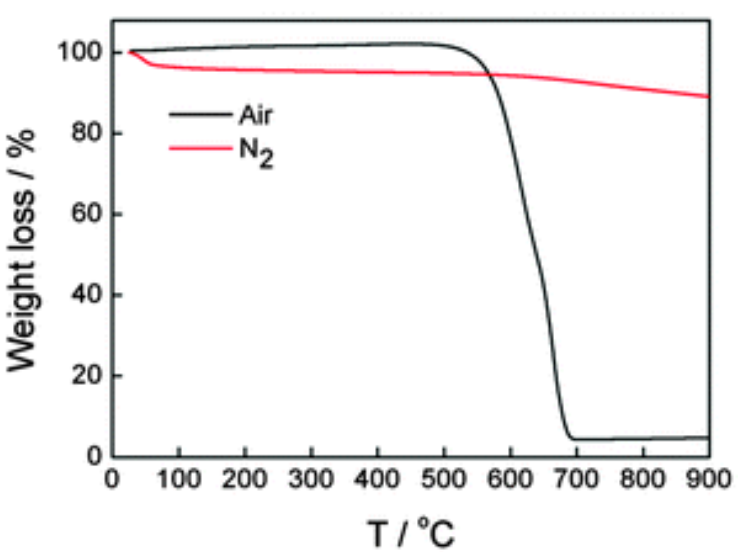

Fig. 1 (A) XRPD, (B) Raman and (C) FT-IR spectra of CS. (D) TGA of CS in air (black line) and a nitrogen atmosphere (red).

Raman spectra have been recorded to disclose the graphitic ordering of CS. Fig. $\underline{1 B}$ shows a representative Raman spectrum with the characteristic $D$ and $G$ bands centred at $1354 \mathrm{~cm}^{-1}$, and $1590 \mathrm{~cm}^{-1}$, respectively. The $D$ band is related to the defects and disorder-induced modes, and the $\mathrm{G}$ band is associated with the in-plane $\mathrm{E}_{2 \mathrm{~g}}$ zonecentre mode, i.e. the vibration of $\mathrm{sp}^{2}$-bonded carbon atoms in a two-dimensional hexagonal lattice. The $I_{\mathrm{D}} / I_{\mathrm{G}}$ ratio is related to graphitic ordering in carbon materials, and a high $I_{0} / I_{G}$ ratio involves a low degree of graphitization (order) in the carbon system, and vice versa. In our sample we measured a $I_{0} / I_{G}$ ratio of $c a .0 .80$, showing a higher degree of graphitization (lower $I_{D} / I_{G}$ ) than most of the other CVD carbon spheres reported to date. ${ }^{10,3,37,3,38} \mathrm{Lower} \mathrm{I}_{\mathrm{D}} / \mathrm{I}_{\mathrm{G}}$ ratios can be achieved in the presence of $\mathrm{MnO}_{2}$ or Ni doping. ${ }^{3940}$ In any case, our results are in accordance with the graphitization trend reported by Nieto-Márquez et al:: graphite > carbon nanotubes/nanofibers > carbon nanospheres $>$ activated carbon. ${ }^{10} \mathrm{~A}$ further comparison of all the synthesized spheres in this work can be found in SI 3 (ESI \pm ). 
FT-IR (Fig. 1C) confirmed the presence of $\mathrm{C}=\mathrm{C}\left(1637 \mathrm{~cm}^{-1}\right)$ and $=\mathrm{C}-\mathrm{H}$ groups $(3100$ and $1080 \mathrm{~cm}^{-1}$, stretching and bending modes, respectively) in the CS, supporting the presence of an $\mathrm{sp}^{2}$ structure. Among these, another band at $c a .1400 \mathrm{~cm}^{-1} \mathrm{can}$ be found, related to the $\mathrm{C}-\mathrm{H}$ bending mode. ${ }^{34}$

Furthermore, thermogravimetric analysis (TGA) and differential thermal analysis (DTA) experiments in air and under a $\mathrm{N}_{2}$ atmosphere up to $900^{\circ} \mathrm{C}$ were carried out. The thermogravimetric profile reveals that CS are stable in air below $530^{\circ} \mathrm{C}$; at $c a .560{ }^{\circ} \mathrm{C}$ they exhibit an abrupt mass loss of $c a .95 \%$ which ended at $700^{\circ} \mathrm{C}$ (Fig. 1D). According to Serp et al. classification of carbon nanoforms based on the maximum of the gasification rate, ${ }^{5}$ our sample is located in the range of carbon nano-spheres with a combustion temperature of $c a .630^{\circ} \mathrm{C}$ depicted in the DTA (see SI 4, ESI \pm ). In turn, the experiment in $\mathrm{N}_{2}$ exhibits a stable thermal behaviour, showing only a weight loss of $c a .10 \%$ when $900{ }^{\circ} \mathrm{C}$ is reached. It is worth noting that in the presence of air, a slight increase of mass (ca. 105\%) is observed just before thermal decomposition; this is attributed to the oxidizing process of graphitic flakes into oxidized graphite. In contrast, this effect is not seen in $\mathrm{N}_{2} \cdot{ }^{34,41}$

After studying the structural properties of the spheres, their morphology was unveiled by means of FESEM and HRTEM, depicting the spherically characteristic shape of CS, with a large quantity of them usually forming aggregates (Fig. 2). The accretion of spheres into clusters of several microns (see SI 5, ESII) was described by Kroto et al. for feeding times higher than $10 \mathrm{~min},{ }^{34}$ or by Pol and co-workers as a result of cooling the system from high temperature to room temperature. ${ }^{35}$ Anyway, it is worth noting that this coalescence is related to the presence of reactive dangling bonds on the surface, which gives them a high surface reactivity. ${ }^{4,10}$ Indeed, the EDAX analysis of the sample revealed a carbon content of 93.2 atomic\% and an oxygen content of $6.8 \%$. These results are corroborated by $\mathrm{C}, \mathrm{H}, \mathrm{N}$ elemental analysis, with a carbon content of $93.3 \%$ (atomic ratio) and $0.4 \%$ of $\mathrm{H}$. No $\mathrm{N}$ was detected. Atomic percentages were also confirmed by XPS (see SI 6.1, ESI 1 ). Furthermore, the XPS spectrum of $\mathrm{C}_{1 \mathrm{~s}}$ clearly exhibits a main peak which can be deconvoluted into $\mathrm{sp}^{2}$ and $\mathrm{sp}^{3}$ components. No trace of the catalyst ( $\mathrm{Ni}$ and $\mathrm{Fe}$ ) was found in the spectra of the CS (SI 6.2, ESII). Finally, the synthesis of carbon spheres at a higher temperature $\left(100{ }^{\circ} \mathrm{C}\right)$ 
depicted a decrease of $c a .1 \%$ in the 0 atomic percentage (a decrease of $c a .11 \%$ of the total oxygen content). The $\mathrm{N}$ signal remains residual (SI 6.1, ESI $\_$).
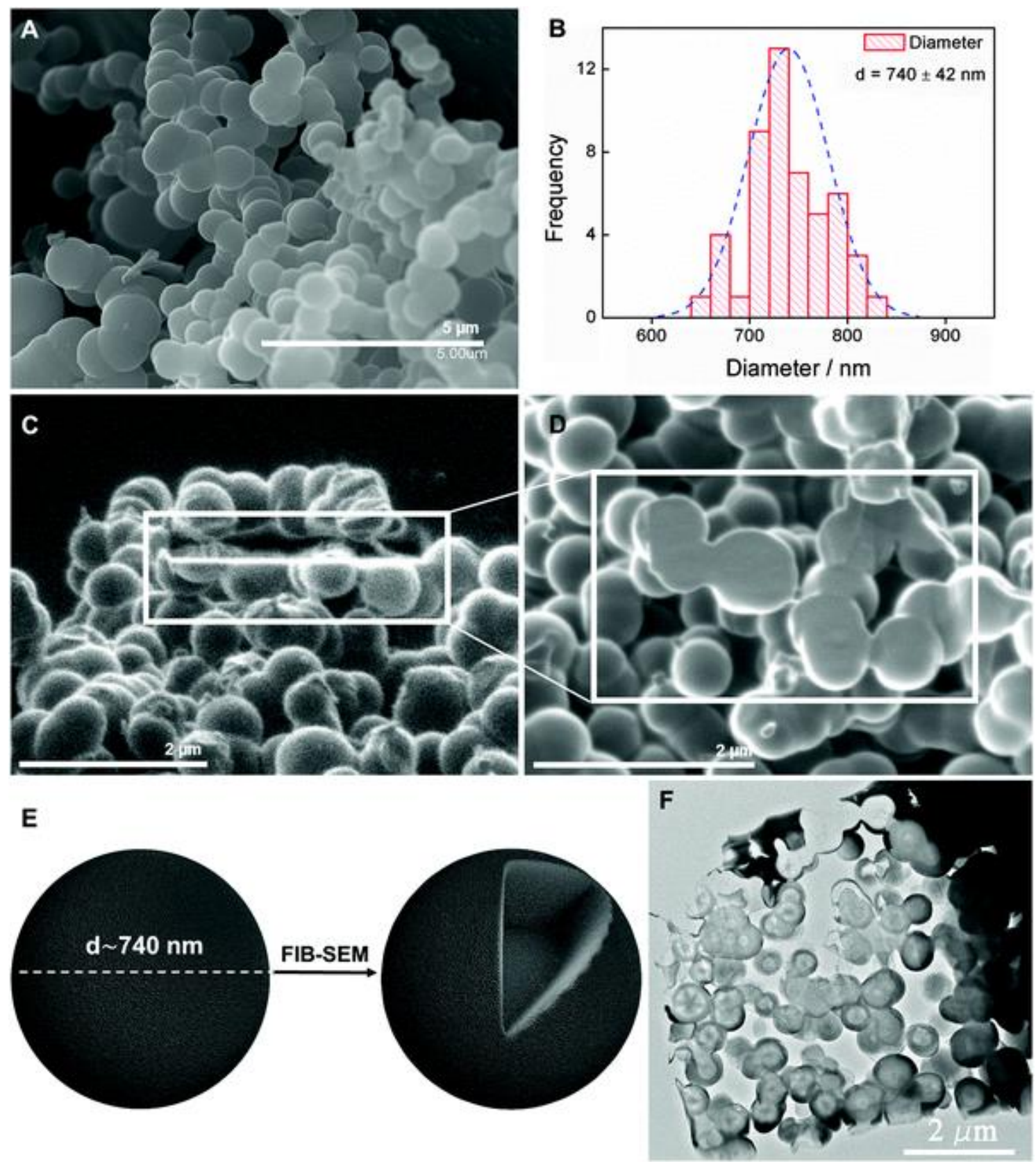

Fig. 2 (A) FESEM image of CS. (B) Histogram displaying the average diameter taken from FESEM images. (C) FIB-SEM image of the milled region, it is remarked within the white square. (D) FIB-SEM cross-section image of the same region of (C) depicting a solid inner structure. (E) Idealized 3D representation of a sphere before and after the FIB, highlighting the solid inner structure. (F) Bright field TEM image of electron-transparent lamella prepared by FIB-SEM. The porosity between the carbon spheres can be clearly seen.

The particle size distribution was determined by measuring 50 spheres in the SEM images, giving rise to an average diameter of $740 \pm 42 \mathrm{~nm}$. This is in sharp contrast with 
the wide range of diameters obtained in the control experiments (see SI 2, ESI \pm ), and highlights the role played by the NiFe-LDH catalytic precursor in controlling the size distribution of the spheres.

In order to determine directly whether the spheres are hollow or not, we have taken advantage of a dual beam focused ion beam-SEM instrument. Precise milling was carried out over several spheres. An example of a typical zone where the focused ion milling process has been applied is shown in Fig. 2C. The cross-section acquired after the milling process supplies detailed information of the specimen subsurface, highlighting the existence of a solid inner structure in the spheres, as depicted in Fig. 2D and $E$.

Furthermore, a lamella thinned down to electron-transparency has been studied by TEM showing the absence of mesopores and porosity between the carbon spheres (vide infra) (Fig. 2F). The preparation of these lamellae is detailed in SI 7 (ESI \pm ).

HRTEM images displayed nearly perfect spherical morphologies with an average diameter of $c a .700-800 \mathrm{~nm}$ (Fig. 3A), disposed usually in a chain-like structure due to the accretion effect. These diameters are in good accordance with the value extracted from FESEM studies. A deeper look into their internal structure revealed a concentric arrangement of the graphitic planes (as demonstrated by HRTEM vide infra). ${ }^{.}$In addition, the distance between the graphitic planes also matches with that calculated from Bragg's equation according to the XRPD spectrum (vide supra). 

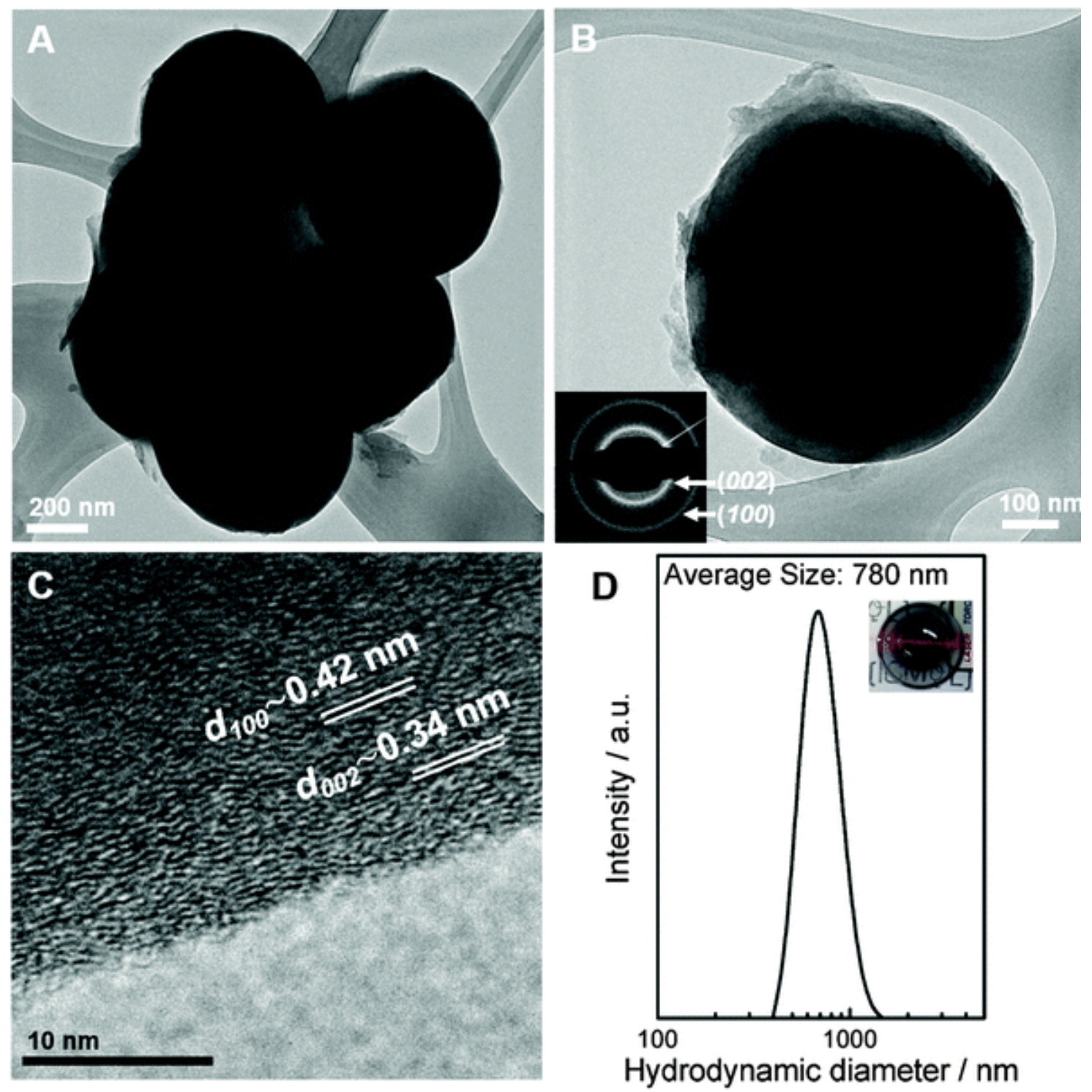

Fig. 3 HRTEM image depicting (A) a cluster of spheres, disposed in a chain-like structure, and (B) a single sphere from the CS sample after a sonication procedure in toluene. The inset in (B) shows the selected area electron diffraction (SAED) pattern displaying two rings corresponding to (002) and (100) planes. (C) High magnification image of the interface from a single sphere, highlighting the (002) and (100) planes and the distance between crystallographic lattices (D). Distribution of sizes extracted from DLS measurements for CS. Its inset shows the visible Tyndall-Faraday effect, resulting from the scattering of the irradiated beam by the particles in a toluene solution.

Interestingly, these chain-like structures can be disassembled by sonication, obtaining relatively stable suspensions of CS exhibiting the characteristic TyndallFaraday effect. Concretely, by suspending the powder in toluene (1 mg of CS in $10 \mathrm{~mL}$ ) and submitting it to bath sonication for $30 \mathrm{~min}$, almost all of the aggregates can be individualized. Fig. $3 \mathrm{~B}$ and $\mathrm{C}$ depict an HRTEM image showing the presence of isolated 
and monodisperse spheres, retaining their intrinsic structure. In their spherical surface, some broken edges can be seen, due to the disaggregation of the clusters after the sonication process. Furthermore, Dynamic Light Scattering (DLS) was carried out with this suspension, in order to further study the size distribution of the sample (Fig. 3D), as well as the possible presence of bigger clusters. We observed that the average hydrodynamic diameter - a complex function that describes a Gaussian distribution - is centred at ca. $780 \mathrm{~nm}$, in good agreement with the electron microscopy observations and the classification proposed by Serp et al. ${ }^{5}$

In order to determine the surface texture of the spheres, $\mathrm{N}_{2}$ adsorption-desorption isotherms were measured. The CS showed a BET surface area value of $16.2 \mathrm{~m}^{2} \mathrm{~g}^{-1}$, indicative of the absence of mesoporosity on its surface, in accordance with previously published studies. ${ }^{10}$ In contrast, carbon spheres with a mesoporous structure depicted larger BET surface areas of $c a .1400-1600 \mathrm{~m}^{2} \mathrm{~g}^{-1} \cdot{ }^{36,37} \mathrm{It}$ is worth saying that this value is slightly higher than that exhibited by the pristine spheres $\left(3.6 \mathrm{~m}^{2} \mathrm{~g}^{-1}\right)$, as a consequence of annealing. In line with that, differential pore volume distribution of the spheres by DFT has been calculated, suggesting the presence of ultramicropores (see SI 8, ESI \pm )..$^{2-44}$

One of the most important fields of application of CS and related nanomaterials is the energy storage and conversion. ${ }^{45,46}$ Among others, supercapacitors (SCs) are attracting increasing attention due to their potential to deliver high power densities in short periods of time with high cyclability and durability. ${ }^{47}$ In this sense, the graphitic character, porosity, conductivity or the presence of oxygen functionalities could greatly affect the properties of SCs, providing us with important information about the presence of oxygen functionalities on the surface.

For comparative purposes, we have tested the electrochemical properties of the CS as electrode materials for SCs in a conventional three-electrode cell. The powdered sample was mixed with acetylene black and poly-vinylidene fluoride at a mass ratio of $80: 10: 10$ with ethanol. The mixture was cast on a $1 \mathrm{~cm}^{2} \mathrm{Ni}$-foam collector, dried and pressed to minimize the loss of the active material during electrochemical testing. The electrochemical properties were measured by means of cyclic voltammetry (CV) using $6 \mathrm{M} \mathrm{KOH} \mathrm{(99.99 \% )} \mathrm{as} \mathrm{the} \mathrm{electrolyte} \mathrm{in} \mathrm{an} \mathrm{applied} \mathrm{voltage} \mathrm{range} \mathrm{of} 0$ to $-1 \mathrm{~V}$ 
(vs. $\mathrm{Ag} / \mathrm{AgCl}$ ). Fig. 4A shows the quasi-rectangular cyclic voltammograms recorded at different scan rates, in which the large areas suggest an appropriate supercapacitive behaviour, although these CS are not porous, indicative of an activated surface. ${ }^{48}$ Moreover, galvanostatic charge-discharge cycling was also measured in order to study the specific capacitance of these CS (ig. 4B). A relatively large discharge capacity of $c a .132 \mathrm{~F} \mathrm{~g}^{-1}$ was obtained for a current density of $1 \mathrm{~A} \mathrm{~g}^{-1}$ (inset in Fig. 4B), overpassing that recently reported for related microporous $\mathrm{CS}^{49}$ a clear indication of the improved graphitic quality of the as-synthesized materials and the presence of an oxidized shell in accordance with the EDAX and elemental analysis results (vide supra). Because the oxygen content has a close relationship with carbonization temperature, the electrochemical properties of CS synthesized at 1000 ${ }^{\circ} \mathrm{C}$ were measured, exhibiting lower values of capacitances as seen in SI 9 (ESI $\perp$ ). This fact can be related to the minor presence of oxygen on the surface of those spheres, as revealed by XPS (SI 6.1, ESII).

\section{A}

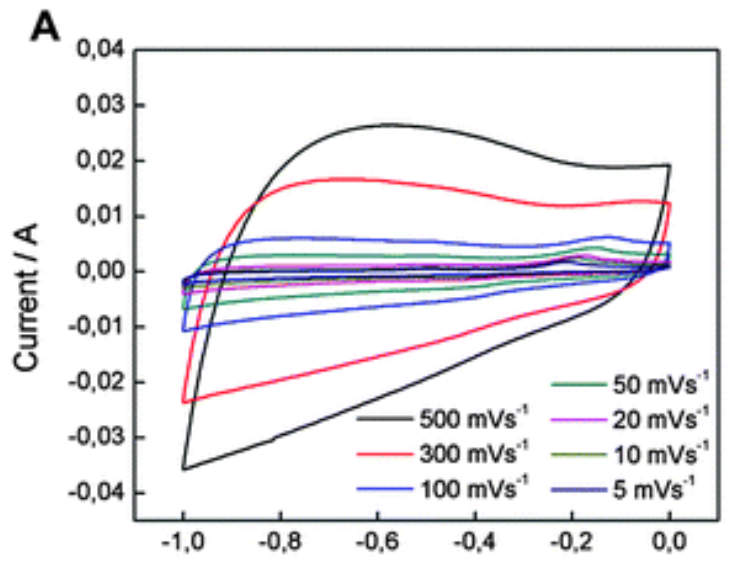

C

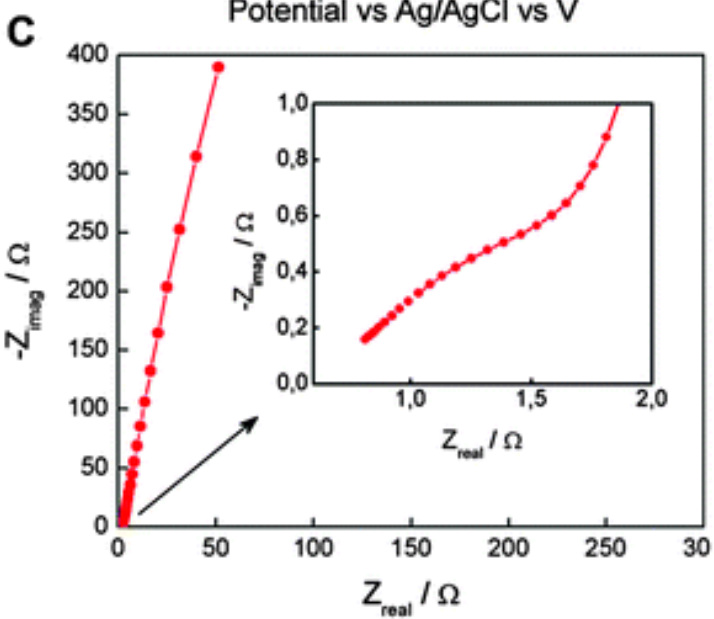

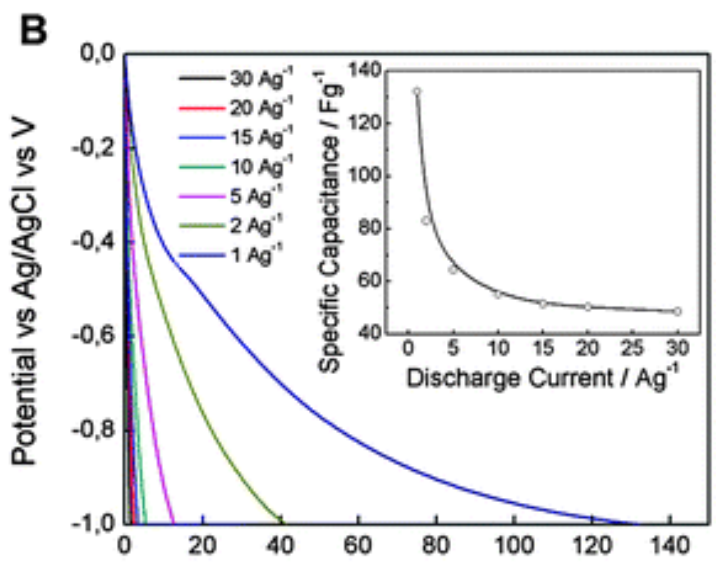

D

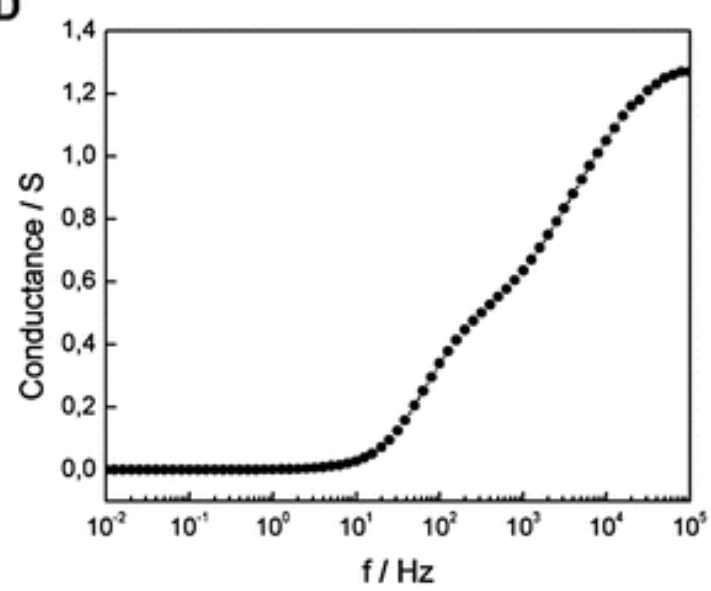


Fig. 4 Electrochemical properties of CS. (A) CV curve at various scan rates in a $6 \mathrm{M} \mathrm{KOH}$ aqueous solution. (B) Galvanostatic discharge curves at different discharge current densities. The inset represents the specific capacitance of the material at different discharge current densities. Electrochemical impedance spectra of CS in a $6 \mathrm{M} \mathrm{KOH}$ electrolyte. (C) Nyquist plots and (D) the response of conductance at different frequencies. The inset shows the high-frequency region.

The potential electrochemical impedance spectroscopy (EIS) shows Nyquist plots with a nearly vertical line, typical of capacitive behaviour. In the inset of Fig. 4 a minor increase in the imaginary part can be seen due to electrolyte resistance. The corresponding conductance shows a typical behaviour, with values close to zero at low frequencies increasing with the increasing frequency, with a maximum of $1.3 \mathrm{~S} .{ }^{50}$

A complete physical characterization of CS has been done measuring the transport properties using the four-probe method. The CS exhibited a nonmetallic behaviour as can be observed in the thermal evolution of resistance (Fig. 5). Fig. 5 shows characteristic non-linear and symmetrical $I-V$ curves for the whole studied temperature range. The non-linearity increases when the temperature is reduced, observing semiconductor behaviour. The electrical conductivity at room temperature is $1.92 \times 10^{-2} \mathrm{~S} \mathrm{~cm}^{-1}$ and it is reduced by four orders of magnitude when the temperature decreases, which is $1.32 \times 10^{-6} \mathrm{~S} \mathrm{~cm}^{-1}$ at $2 \mathrm{~K}$ (Fig. 5). Furthermore, the conductivity matches perfectly with other values reported in the literature for CS. ${ }^{16,17}$

A

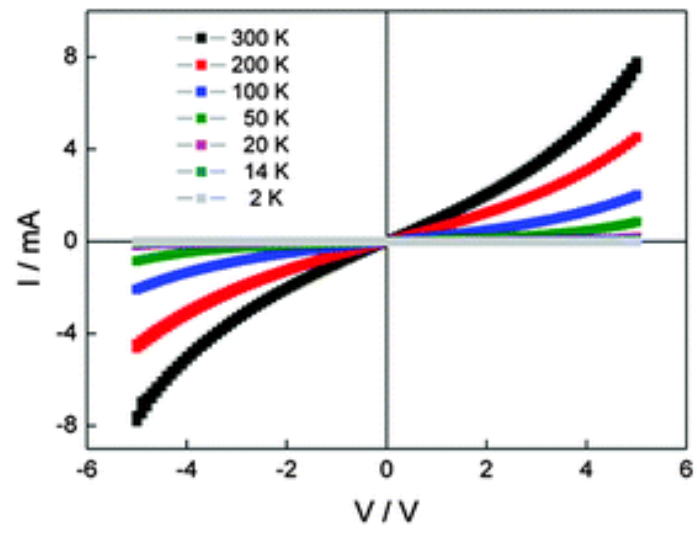

B

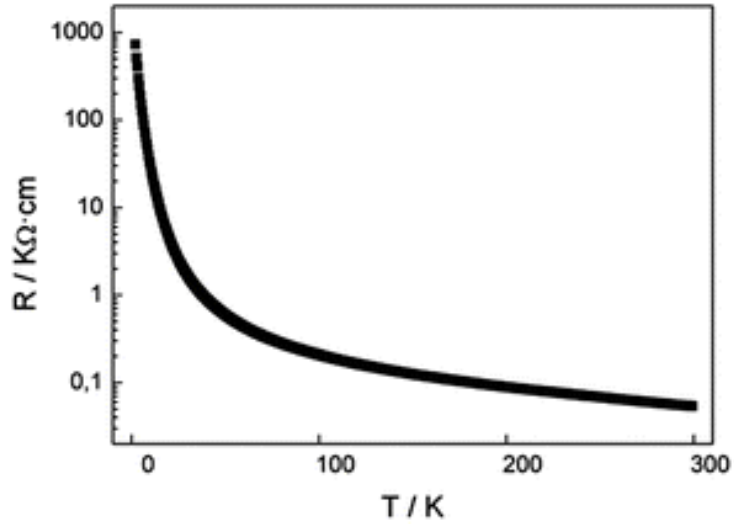

Fig. 5 (A) $I-V$ curves for a temperature range from $300 \mathrm{~K}$ to $2 \mathrm{~K}$. The electrical conductivity at room temperature is $1.92 \times 10^{-2} \mathrm{~S} \mathrm{~cm}^{-1}$. (B) Thermal resistance dependence of the CS.

Studies dealing with the electrical properties of CS are very scarce. To the best of our knowledge, the first work that has measured the electrical properties of the CS is from Coville et al., ${ }^{16,17}$ where they studied the electrical transport mechanisms in nitrogen and boron-doped carbon spheres. These studies show that the dominant 
conduction mechanism in pristine CS is Mott variable range hopping (Mott VRH). In our case, this model provides a good description of the thermal dependence of electrical conductivity in the 300-2 $\mathrm{K}$ temperature range (Fig. SI 10, ESII). Thus, the experimental behaviour is well fitted by the expression $\bigotimes(T)=\bigotimes_{0} \cdot \exp \left[-\left(T_{0} / T\right)^{n}\right]$, with $n=$ $1 / 4$. The VRH model of conductivity represents a phonon-assisted mechanism of charge transport and it is normally associated with amorphous semiconductors, semiconductor glasses ${ }^{51,52}$ and more recently with amorphous carbon. ${ }^{1}$

We also measured the magnetoresistance (MR) properties of CS in the applied fields of $8 \mathrm{~T}$ in a PPMS system, revealing an intrinsic magnetoresistance (MR) of $c a$. $72 \%$ at low temperatures (Fig. 6). MR is calculated as MR (\%) $=[R(H)-R(0) / R(0)] \times 100$, where $R(H)$ is the resistivity under the magnetic field $B$ and $R(0)$ is the resistivity without any magnetic field. As long as we increase the temperature, the MR falls dramatically until no signal is observed above $100 \mathrm{~K}$.

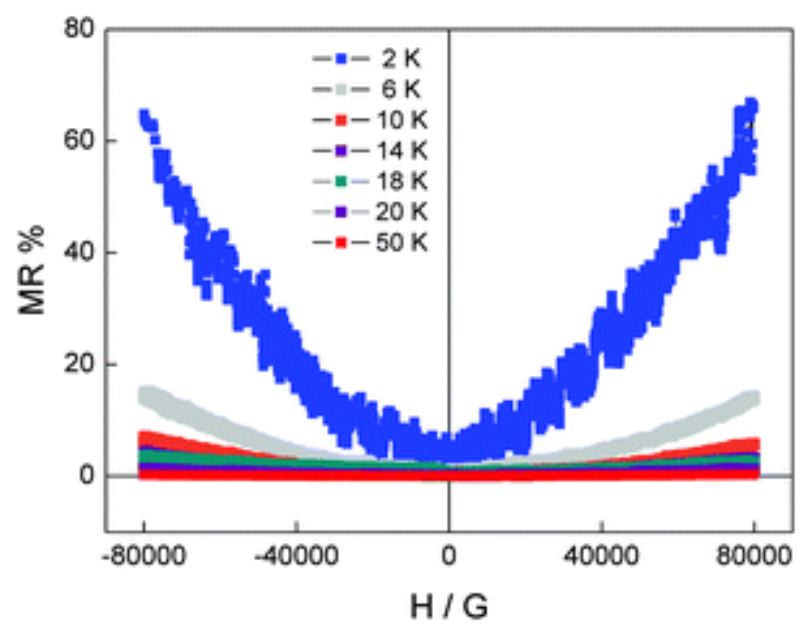

Fig. 6 MR dependence on the temperature of the CS.

Fig. 6 shows a positive and non-saturated MR that decreases dramatically when the temperature increases for all the temperature range studied (from $50 \mathrm{~K}$ to $2 \mathrm{~K}$ ). This positive, non-saturated MR behavior has also been found for other crystalline and amorphous nanocarbons and carbon composites. ${ }^{20-25}$ All the experimental data of the Fig. 6 were fitted with the relation of MR $\propto\left(\bigotimes_{0} \cdot \nabla \cdot|H|\right)^{2}$ (Fig. SI 10, ESI \pm ). The quadratic term is related to the ordinary $M R(O M R)^{20}$ due to the curving of electron trajectories in the carbon plane, which results in a positive MR, and has a quadratic

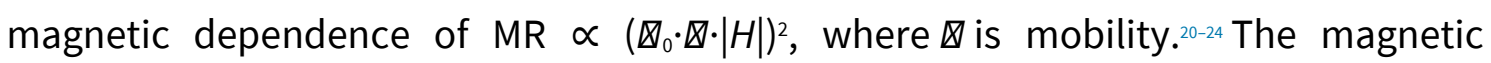


measurements revealed a very small magnetic phase in the CS (Fig. SI 11, ESI 1 ). This magnetic phase probably comes from a small rest of the catalyst. However, the XPS analysis of CS showed the absence of the catalyst on the sample surface, highlighting the residual character of the catalyst present in the CS (Fig. SI 6.2, ESI \pm ). Therefore, the influence of the magnetic rest of the catalyst in the MR is very weak, and almost negligible.

Finally, the dramatic temperature dependence of the MR (Fig. SI 12, ESI \pm ) is an indication of the predominant contribution of the OMR to the MR observed in the CS. These results pave the way for using CS not only as a reinforcing agent of polymers, but also as electronically active building blocks in the design of hybrid multifunctional composites.

\section{Conclusions}

Carbon spheres with a diameter of $c a .740 \mathrm{~nm}$ have been synthesized in gram-scale proportions by means of a CVD method using NiFe-LDHs as catalyst precursors. Control experiments revealed that the presence of the $\mathrm{FeNi}_{3}$ catalyst allows us to control the size distribution, giving rise to a monodisperse sample. XRPD and Raman spectroscopy demonstrate their graphitic nature, with an $I_{D} / I_{G}$ ratio of $c a .0 .80$, therefore exhibiting a higher degree of graphitization than other spheres reported to date. Moreover, these CS can be individualized in toluene by means of sonication, beating the accretion effects. Furthermore, FIB-SEM experiments show that the inner structure of the spheres is solid and consists of concentric graphitic planes. Concerning their surface properties, nitrogen adsorption, XPS and electrochemical testing revealed the presence of redox functionalities on their surface behaving as appropriated electrode materials for supercapacitors despite their intrinsic nonmesoporous nature.

Finally, their transport properties have also been studied, exhibiting a semiconducting behaviour at low temperatures and a giant magnetoresistance of $c a .72 \%$ at $2 \mathrm{~K}$ and a voltage of $3 \mathrm{~V}$. The MR was strongly dependent on the temperature and principally has a quadratic dependence, following an ordinary MR behaviour. These CS are promising candidates for polymer reinforcement and as building blocks for preparing more complex hybrid composites.

\section{Acknowledgements}


Financial support from the EU (FET-OPEN 2D-INK. Grant agreement 648786), the Spanish MINECO (Project MAT2014-56143-R), the Junta de Andalucía (PAI research group TEP-946 INNANOMAT) and the Generalitat Valenciana (Prometeo and ISIC-Nano Programs) is gratefully acknowledged. Co-funding from UE is also acknowledged. TEM measurements were carried out at DME-SCCYT-UCA. We also acknowledge E. Bandiello for his help with the EIS experiments. We thank the Universidad de Valencia for support from VLC/CAMPUS and INNCIDE program, and for a predoctoral grant (to J. A. C.). G. A. thanks the EU for a Marie Curie Fellowship (FP7/2013-IEF-627386).

\section{References}

1. H. W. Kroto, J. R. Heath, S. C. O'Brien, R. F. Curl and R. E. Smalley, Nature, 1985, 318, 162-163 CrossRef CAS.

2. J. L. Delgado, M. Herranz and N. Martín, J. Mater. Chem., 2008, 18, 14171426 RSC.

3. A. W. Francis, Ind. Eng. Chem., 1931, 23, 612-617 CrossRef CAS.

4. Z. C. Kang and Z. L. Wang, J. Phys. Chem., 1996, 100, 5163-5165 CrossRef CAS.

5. P. Serp, R. Feurer, P. Kalck, Y. Kihn, J. L. Faria and J. L. Figueiredo, Carbon, 2001, 39, 621-626 CrossRef CAS.

6. M. Inagaki, Solid State Ionics, 1996, 86, 833-839 CrossRef.

7. M. Inagaki, Carbon, 1997, 35, 711-713 CrossRef CAS.

8. Y. Xia, B. Gates, Y. Yin and Y. Lu, Adv. Mater., 2000, 12, 693-713 CrossRef CAS.

9. A. A. Deshmukh, S. D. Mhlanga and N. J. Coville, Mater. Sci. Eng., R, 2010, 70, 128 CrossRef.

10. A. Nieto-Márquez, R. Romero, A. Romero and J. L. Valverde, J. Mater. Chem., 2011, 21, 1664-1672 RSC.

11. Carbon materials for catalysis, ed. P. Serp and J. L. Figueiredo, John Wiley \& Sons, Hoboken, N.J, 2009 Search PubMed.

12. N. Fleischer, M. Genut and A. Gorodnev, Am. Ceram. Soc. Bull., 2006, 85, 2728 CAS.

13. X. He, F. Wu and M. Zheng, Diamond Relat. Mater., 2007, 16, 311315 CrossRef CAS.

14. Y. Z. Jin, Y. J. Kim, C. Gao, Y. Q. Zhu, A. Huczko, M. Endo and H. W. Kroto, Carbon, 2006, 44, 724-729 CrossRef CAS.

15. F. P. Hu, Z. Wang, Y. Li, C. Li, X. Zhang and P. K. Shen, J. Power Sources, 2008, 177, 61-66 CrossRef CAS.

16. W. P. Wright, V. D. Marsicano, J. M. Keartland, R. M. Erasmus, S. M. A. Dube and N. J. Coville, Mater. Chem. Phys., 2014, 147, 908-914 CrossRef CAS.

17. K. C. Mondal, A. M. Strydom, Z. Tetana, S. D. Mhlanga, M. J. Witcomb, J. Havel, R. M. Erasmus and N. J. Coville, Mater. Chem. Phys., 2009, 114, 973977 CrossRef CAS. 
18. Y. Z. Jin, C. Gao, H. W. Kroto and T. Maekawa, Macromol. Rapid Commun., 2005, 26, 1133-1139 CrossRef CAS.

19. H. Ismail, P. K. Freakley and E. Sheng, Eur. Polym. J., 1995, 31, 10491056 CrossRef CAS.

20. H. Gu, X. Zhang, H. Wei, Y. Huang, S. Wei and Z. Guo, Chem. Soc. Rev., 2013, 42, 5907-5943 RSC.

21. C. Bosch-Navarro, F. Busolo, E. Coronado, Y. Duan, C. Martí-Gastaldo and H. Prima-Garcia, J. Mater. Chem. C, 2013, 1, 4590-4598 BSC.

22. J. Wang, X. Zhang, C. Wan, J. Vanacken and V. V. Moshchalkov, Carbon, 2013, 59, 278-282 CrossRef CAS.

23. Z.-M. Liao, H.-C. Wu, S. Kumar, G. S. Duesberg, Y.-B. Zhou, G. L. W. Cross, I. V. Shvets and D.-P. Yu, Adv. Mater., 2012, 24, 1862-1866 CrossRef CAS PubMed.

24. Y.-B. Zhou, B.-H. Han, Z.-M. Liao, H.-C. Wu and D.-P. Yu, Appl. Phys. Lett., 2011, 98, 222502 CrossRef.

25. J. Zhu, Z. Luo, S. Wu, N. Haldolaarachchige, D. P. Young, S. Wei and Z. Guo, J. Mater. Chem., 2012, 22, 835-844 RSC.

26. G. Abellán, C. Martí-Gastaldo, A. Ribera and E. Coronado, Acc. Chem. Res., 2015, 48, 1601-1611 CrossRef PubMed.

27. G. Abellán, E. Coronado, C. Martí-Gastaldo, A. Ribera and J. F. SánchezRoyo, Chem. Sci., 2012, 3, 1481-1485 RSC.

28. G. Abellán, J. A. Carrasco, E. Coronado, J. P. Prieto-Ruiz and H. PrimaGarcía, Adv. Mater. Interfaces, 2014, 1, 1400184 Search PubMed.

29. G. Abellán, J. G. Martínez, T. F. Otero, A. Ribera and E. Coronado, Electrochem. Commun., 2014, 39, 15-18 CrossRef.

30. G. Abellán, E. Coronado, C. Martí-Gastaldo, A. Ribera and T. F. Otero, Part. Part. Syst. Charact., 2013, 853-863 Search PubMed.

31. G. Abellán, E. Coronado, C. Martí-Gastaldo, E. Pinilla-Cienfuegos and A. Ribera, J. Mater. Chem., 2010, 20, 7451-7455 BSC.

32. R. Langford and C. Clinton, Micron, 2004, 35, 607-611 CrossRef CAS PubMed.

33. Y. Li, J. Chen, Q. Xu, L. He and Z. Chen, J. Phys. Chem. C, 2009, 113, 10085$10089 \underline{\text { CAS. }}$.

34. Y. Z. Jin, C. Gao, W. K. Hsu, Y. Zhu, A. Huczko, M. Bystrzejewski, M. Roe, C. Y. Lee, S. Acquah, H. Kroto and D. R. M. Walton, Carbon, 2005, 43, 19441953 CrossRef CAS.

35. V. G. Pol, M. Motiei, A. Gedanken, J. Calderon-Moreno and M. Yoshimura, Carbon, 2004, 42, 111-116 CrossRef CAS.

36. L. C. Yang, Y. Shi, Q. S. Gao, B. Wang, Y. P. Wu and Y. Tang, Carbon, 2008, 46, 1816-1818 CrossRef CAS.

37. A. Nieto-Márquez, D. Toledano, J. C. Lazo, A. Romero and J. L. Valverde, Appl. Catal., A, 2010, 373, 192-200 CrossRef.

38. H. Qian, F. Han, B. Zhang, Y. Guo, J. Yue and B. Peng, Carbon, 2004, 42, 761766 CrossRef CAS.

39. M. Liu, L. Gan, W. Xiong, Z. Xu, D. Zhu and L. Chen, J. Mater. Chem. A, 2014, 2, 2555-2562 CAS. 
40. M. Liu, L. Gan, W. Xiong, F. Zhao, X. Fan, D. Zhu, Z. Xu, Z. Hao and L. Chen, Energy Fuels, 2013, 27, 1168-1173 CrossRef CAS.

41. Y. J. Kim, M. I. Kim, C. H. Yun, J. Y. Chang, C. R. Park and M. Inagaki, J. Colloid Interface Sci., 2004, 274, 555-562 CrossRef CAS PubMed.

42. W. Li, D. Chen, Z. Li, Y. Shi, Y. Wan, G. Wang, Z. Jiang and D. Zhao, Carbon, 2007, 45, 1757-1763 CrossRef CAS.

43. D. Fujikawa, M. Uota, T. Yoshimura, G. Sakai and T. Kijima, Chem. Lett., 2006, 35, 432-433 CrossRef CAS.

44. M. Liu, J. Qian, Y. Zhao, D. Zhu, L. Gan and L. Chen, J. Mater. Chem. A, 2015, 3, $11517-11526$ CAS

45. M. Liu, X. Ma, L. Gan, Z. Xu, D. Zhu and L. Chen, J. Mater. Chem. A, 2014, 2, 1710717114 CAS.

46. D. Zhu, Y. Wang, L. Gan, M. Liu, K. Cheng, Y. Zhao, X. Deng and D. Sun, Electrochim. Acta, 2015, 158, 166-174 CrossRef CAS.

47. F. Beguin and E. Frackowiak, Supercapacitors: Materials, Systems and Applications, John Wiley \& Sons, 2013 Search PubMed.

48. X. Ma, L. Gan, M. Liu, P. K. Tripathi, Y. Zhao, Z. Xu, D. Zhu and L. Chen, J. Mater. Chem. A, 2014, 2, 8407-8415 CAS.

49. W. Yang, Y. Feng, D. Xiao and H. Yuan, Int. J. Energy Res., 2015, 39, 805811 CrossRef CAS.

50. Y. Gao, Y. S. Zhou, M. Qian, X. N. He, J. Redepenning, P. Goodman, H. M. Li, L. Jiang and Y. F. Lu, Carbon, 2013, 51, 52-58 CrossRef CAS.

51. N. F. Mott, Philos. Mag., 1969, 19, 835-852 CrossRef CAS.

52. N. F. Mott and M. Kaveh, Adv. Phys., 1985, 34, 329-401 CrossRef CAS. 